\title{
Consenso chileno SER-SOCHINEP para el manejo del asma en el preescolar ${ }^{\#}$
}

\author{
ANA MARÍA HERRERA G.* (COORDINADORA), SELIM ABARA E.**, CECILIA ÁLVAREZ G.**, \\ CLAUDIA ASTUDILLO M.***, RAÚl CORRALES V.**, EVELYN CHALA C.****, \\ TATIANA ESPINOZA P.*****, ANDRÉS KOPPMANN A.******, VIVIANA LEZANA S.*******, \\ ROSSANA MAMANI J.*, MARÍA ANGÉLICA PÉREZ H.* y CRISTINA PIERRY V.**
}

\section{Chilean consensus on management of asthma in preschool children}

Preschool wheezing is one of the most common presenting symptoms in pediatric practice. The vast majority of these patients present wheeze triggered by viral respiratory infections and only a minority of them will have asthma in school age. The evaluation of these children begin with a detailed clinical history and physical examination and standard laboratory tests to rule out secondary causes of wheezing. The decision for controlling therapy will depend on the frequency and severity of the wheezing episodes, but should always be considered as a treatment trial, avoiding prolonged periods of time. It is recommended to start with low doses of inhaled corticosteroids, evaluating treatment effect with a close follow up. Inhaled bronchodilators remain the treatment of choice in acute exacerbations of wheezing, systemic corticosteroids should be reserved for severe exacerbations in hospitalized patients.

Key words: Asthma, preschool, preschool wheezing disorder, episodic viral wheeze, multiple trigger wheeze.

\section{Resumen}

Las sibilancias recurrentes del preescolar es una condición frecuente a la que se ve enfrentado el pediatra. La gran mayoría de estos pacientes presentan sibilancias en relación a infecciones respiratorias virales y sólo una minoría de ellos tendrá diagnóstico de asma en la edad escolar. El enfrentamiento adecuado de estos niños se inicia con una detallada anamnesis y examen físico para descartar causas secundarias, lo que se complementa con algunos exámenes de laboratorio. La decisión de iniciar tratamiento de mantención dependerá principalmente de la frecuencia y gravedad de los episodios de sibilancias. Este tratamiento debe ser considerado como una prueba terapéutica, evitando

\#Documento elaborado por las Comisiones de Asma Pediátrico de la Sociedad Chilena de Enfermedades Respiratorias y Sociedad Chilena de Neumología Pediátrica.

Considerando la importancia de este tema, la Sociedad Chilena de Enfermedades Respiratorias y la Sociedad Chilena de Neumología Pediátrica han acordado publicar este consenso conjuntamente en sus órganos oficiales Revista Chilena de Enfermedades Respiratorias y Revista Chilena de Neumología Pediátrica.

Declaración de conflicto de intereses: Los Drs. A. M. Herrera G., S. Abara E., C. Álvarez G, C. Astudillo M., R. Corrales V., E. Chala C., T. Espinoza P., A. Koppmann A., V. Lezana S., R. Mamani J. y C. Pierry V. declaran no presentar conflicto de intereses. La Dra. M. Angélica Pérez H. recibió honorarios por concepto de docencia de parte del laboratorio GSK.

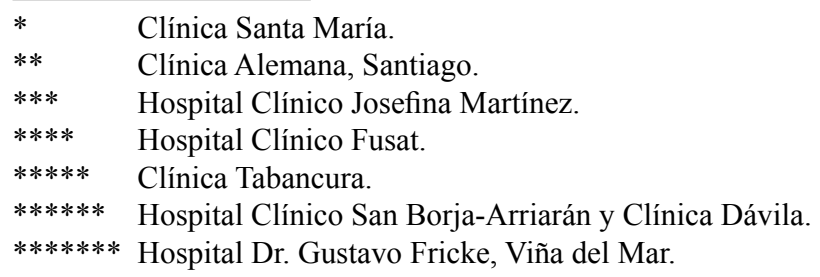


su mantención por tiempos prolongados. Se recomienda el uso de corticoides inhalados en dosis bajas y realizar un seguimiento estrecho del paciente para objetivar la respuesta. En el caso de los episodios agudos los broncodilatadores son el tratamiento de elección, mientras que los corticoides sistémicos debieran reservarse sólo para los casos severos o que requieren hospitalización.

Palabras clave: Asma, preescolar, sibilancias recurrentes, sibilancias inducidas por virus, sibilancias multigatilladas.

\section{Introducción}

Las sibilancias recurrentes del preescolar es una condición muy frecuente en pediatría. Un tercio de los niños presentan al menos un episodio de sibilancias antes de los tres años de vida y $50 \%$ antes de los seis. Sin embargo, la presencia de sibilancias recurrentes no es sinónimo de asma. Muchos niños pequeños pueden presentar sibilancias durante las infecciones respiratorias virales pero sólo una minoría de ellos va a tener diagnóstico de asma en la edad escolar ${ }^{1-4}$. Las sibilancias recurrentes corresponden a una entidad muy heterogénea, con distintos mecanismos fisiopatológicos de base, por lo que el diagnóstico de asma en el pre-escolar plantea un gran desafío para el médico tratante. Además en este grupo etario no contamos con medidas objetivas de evaluación de la función pulmonar ni biomarcadores $^{4}$, a pesar de lo cual es muy importante intentar distinguir dentro del grupo de sibilantes recurrentes cuáles corresponden a los posibles asmáticos, de manera de indicar tratamiento de mantención sólo a este subgrupo de pacientes, evitando su uso en los que no lo requieren. Es así como se han diseñado distintos índices predictores de asma derivados de estudios de cohortes, siendo el más utilizado el de Castro Rodríguez ${ }^{5}$. Existen diferentes fenotipos de sibilancias recurrentes de acuerdo a la Cohorte de Tucson ${ }^{6}$. Los sibilantes transitorios $(20 \%)$ presentaban sibilancias en los primeros tres años de vida, pero dejaban de sibilar a los 6 , tenían una función pulmonar disminuida al nacer y como factor de riesgo el tabaquismo materno durante el embarazo. Los sibilantes persistentes (14\%) iniciaban sibilancias en los primeros tres años y seguían sibilando a los seis, presentaban una función pulmonar normal al nacer, pero que disminuía hacia la edad escolar y tenían antecedentes familiares y personales de atopía. Este grupo era el que presentaba mayor riesgo de desarrollar asma. Por último los sibilantes tardíos (15\%) iniciaban sibilancias después de los 3 años y continuaban sibilando a los seis, tenían antecedentes de atopía personal y familiar y a diferencia del grupo anterior tenían función pulmonar normal. La Cohorte $\mathrm{ALSPAC}^{7}$ confirmó los fenotipos descritos en la
Cohorte de Tucson y agrega dos fenotipos más, uno de sibilantes de inicio intermedio y otro de sibilantes precoces de evolución prolongada. El primero iniciaba sibilancias después de los 18 meses y estaba fuertemente relacionado con atopía y desarrollo posterior de asma bronquial $\mathrm{y}$ el segundo iniciaba sibilancias precozmente remitiendo a los 69 meses. Este último grupo se comportaba como los transitorios de la Cohorte de Tucson pero dejaba de presentar sibilancias en forma más tardía.

\section{Diagnóstico de asma en el preescolar}

El diagnóstico de asma en esta edad es fundamentalmente clínico y debe plantearse en todo niño que presente 3 o más episodios de obstrucción bronquial en un año. Desde el punto de vista clínico las sibilancias recurrentes del preescolar pueden presentarse de acuerdo a dos patrones diferentes ${ }^{8,9}$ :

\section{a) Sibilancias episódicas virales}

Episodios recurrentes de obstrucción bronquial de gravedad y duración variables gatillados por infecciones virales. Este patrón se caracteriza por tener períodos intercríticos asintomáticos, presentándose preferentemente en los meses de otoño e invierno.

\section{b) Sibilancias multigatilladas}

Episodios recurrentes de obstrucción bronquial desencadenados por infecciones virales y también por otros estímulos no infecciosos como exposición a humo de tabaco, aire frío, alergenos, ejercicio, llanto, risa, niebla, etc. Este fenotipo puede asociarse más fuertemente a asma bronquial.

Es importante señalar que esta clasificación tiene algunos inconvenientes. El primero es que estos fenotipos no son estables en el tiempo, pudiendo cambiar en un mismo niño y el segundo es que no considera la frecuencia y gravedad de los episodios, elementos muy importantes a tener en cuenta al momento de decidir instaurar o no una terapia de mantención. En la actualidad se considera que la frecuencia y la severidad de los episodios de sibilancias son mejores predictores 
del pronóstico a largo plazo que el patrón temporal de sibilancias (sibilancias inducidas por virus o multigatilladas) en la edad preescolar ${ }^{10}$.

\section{Diagnóstico diferencial}

Siempre deben descartarse causas secundarias de obstrucción bronquial para lo cual es fundamental una historia clínica detallada y un examen físico acucioso $^{11,12}$ (Tablas 1 y 2). El diagnóstico clínico debe complementarse con los siguientes exámenes de laboratorio: radiografía de tórax anteroposterior y lateral, test de sudor, hemograma y cuantificación de inmunoglobulinas. Otro tipo de exámenes dependerá de la sospecha diagnóstica.

Una vez descartadas las causas secundarias nos quedamos con dos opciones en cuanto a etiología. La primera es que el paciente tenga una vía aérea más estrecha que lo predisponga anatómicamente a tener episodios de obstrucción

Tabla 1. Enfermedades que producen sintomatología respiratoria recurrente ${ }^{11,12}$

\begin{tabular}{|c|c|}
\hline Según sistema comprometido & Etiologías \\
\hline Enfermedades rinosinusales & $\begin{array}{l}\text { Rinosinusitis } \\
\text { Descarga posterior }\end{array}$ \\
\hline Enfermedades digestivas & $\begin{array}{l}\text { Aspiraciones recurrentes } \\
\text { Trastorno de deglución } \\
\text { Fístula tráqueo-esofágica }\end{array}$ \\
\hline Enfermedades obstructivas del árbol tráqueo-bronquial & $\begin{array}{l}\text { Cuerpo extraño } \\
\text { Malformación de la vía aérea } \\
\text { Anillos traqueales completos, } \\
\text { Tráqueo-broncomalacia } \\
\text { Tumor endobronquial } \\
\text { Anillo vascular }\end{array}$ \\
\hline Enfermedades cardiovasculares & $\begin{array}{l}\text { Cardiopatía congénita } \\
\text { Insuficiencia cardíaca } \\
\text { Anillos vasculares }\end{array}$ \\
\hline Enfermedades bronco-pulmonares & $\begin{array}{l}\text { Displasia broncopulmonar } \\
\text { Fibrosis quística } \\
\text { Disquinesia ciliar primaria } \\
\text { Bronquiectasia } \\
\text { Bronquiolitis obliterante }\end{array}$ \\
\hline Enfermedades infecciosas e inmunológicas & $\begin{array}{l}\text { Inmunodeficiencias } \\
\text { Aspergilosis broncopulmonar alérgica } \\
\text { Anafilaxia }\end{array}$ \\
\hline
\end{tabular}

Tabla 2. Cuando sospechar otras patologías

\begin{tabular}{|l|}
\hline - Síntomas presentes desde período neonatal \\
- Antecedentes familiares de enfermedad pulmonar crónica \\
- Estridor, laringitis recurrente, antecedentes de intubación traqueal. Voz o llanto anormal \\
- Tos productiva y/o persistente \\
- Signología pulmonar o imágenes radiográficas con alteraciones localizadas \\
- Compromiso nutricional \\
- Hipocratismo digital, pólipos nasales o signos de insuficiencia cardíaca \\
- Antecedentes o certificación de cianosis sin signos de insuficiencia respiratoria \\
- Infecciones recurrentes ( $\geq 2$ neumonía, $\geq 2$ sinusitis, $\geq 9$ otitis en 1 año) \\
\hline
\end{tabular}


bronquial frente a infecciones respiratorias de origen viral, situación que mejorará en la medida que crezca. La segunda opción es que sea realmente asmático, en cuyo caso se justificará instaurar un tratamiento de mantención. Hacer la diferenciación entre estos dos grupos no es fácil. Uno de los elementos clínicos de utilidad es indagar acerca de la presencia de tos nocturna o gatillada por ejercicio, risa, aire frío o llanto, en ausencia de infección respiratoria, lo que orienta al diagnóstico de asma. Junto con lo anterior es útil preguntar acerca de la presencia de asma bronquial en familiares de primer grado y demostrar la presencia de atopía a través de un test cutáneo o de la determinación de IgE específica para distintos alergenos. Con respecto al uso de las pruebas de función pulmonar en el preescolar, debemos decir que no existe suficiente evidencia para utilizarlas en forma rutinaria ${ }^{13,14}$. Sin embargo, en laboratorios especializados podría ser una herramienta útil al demostrar una respuesta broncodilatadora positiva. Las pruebas de hiperreactividad bronquial como el test de metacolina y el test de ejercicio no están estandarizadas en este grupo etario y la medición de óxido nítrico exhalado $\left(\mathrm{F}_{\mathrm{E}} \mathrm{NO}\right)$ por ahora sólo se utiliza con fines de investigación.

\section{Tratamiento}

En la actualidad se considera que el tratamiento controlador debiera iniciarse en todo preescolar que presente episodios de sibilancias muy frecuentes o severos y en los que tienen síntomas frecuentes entre las crisis (la mayoría de los días de la semana y que responden a broncodilatador $)^{10}$. Los medicamentos más utilizados como tratamiento de mantención son los corticoides inhalados y los antileucotrienos (Montelukast), siendo descritos en la literatura varios esquemas de administración. El estado actual de la evidencia no permite establecer cuáles son los niños que responderán a corticoides inhalados, a Montelukast o a ninguno de ellos ${ }^{10}$. Las terapias combinadas (corticoides inhalados con agonistas $\beta-2$ adrenérgicos de acción prolongada) están aprobadas para su uso sólo en mayores de 4 años, ya que no hay estudios de eficacia y seguridad bajo esta edad. Este tipo de tratamiento sólo debería ser indicado por especialistas. Dado que en los preescolares el diagnóstico de asma no es certero, es importante enfatizar que los distintos tratamientos disponibles deben ser considerados siempre como una prueba terapéutica, no debiendo ser mantenidos por tiempos prolongados.

\section{a) Corticoides inhalados}

Los corticoides inhalados a esta edad deben ser utilizados en dosis pequeñas de acuerdo a lo recomendado por las Guías GINA: Budesonida $200 \mu \mathrm{g}$ al día o Fluticasona $100 \mu \mathrm{g}$ al día administrados con aerocámara. Se recomienda que la prueba terapéutica con estos medicamentos dure 3 meses. La utilización de los corticoides inhalados como tratamiento de mantención está bien sustentada por la evidencia científica ${ }^{15-18}$. En estos estudios se observó que el uso de corticoides inhalados se asocia a reducción significativa de las exacerbaciones, aumento de los días libres de síntomas, menor puntaje de síntomas y menor uso de salbutamol en comparación con el grupo tratado con placebo. En un meta-análisis se demostró que la respuesta satisfactoria al uso de corticoides inhalados en este grupo de pacientes es independiente del fenotipo y de la condición atópica ${ }^{10,16}$. Dentro del grupo de niños que fue tratado con corticoides inhalados los que más se beneficiaban en términos de menor uso de corticoides orales, consultas al Servicio de Urgencia y uso de salbutamol, fueron los niños que tenían antecedentes de consulta a Servicio de Urgencia el año previo, sensibilización alérgica, más síntomas basales (menos de $80 \%$ días libres de síntomas) y los de género masculino y raza blanca ${ }^{18}$. Existen algunas publicaciones que reportan el uso de corticoides inhalados en forma intermitente frente a los primeros síntomas de una infección respiratoria alta utilizando dosis de $1.500 \mu \mathrm{g}$ al día de Fluticasona ${ }^{19}$. Este esquema logró demostrar una reducción de $50 \%$ en la frecuencia de exacerbaciones que requerían del uso de corticoides sistémicos en el grupo tratado en comparación con el grupo placebo. Sin embargo, no hubo diferencias en la frecuencia de hospitalización y además la ganancia en peso y talla en los niños que recibieron esta megadosis de corticoides inhalados fue significativamente menor. No se recomienda adoptar este tipo de estrategia en la práctica clínica diaria, dado el potencial sobreuso de corticoides inhalados en un grupo etario con alta prevalencia de infecciones virales y con riesgo de efectos adversos a largo plazo.

\section{b) Antileucotrienos}

Otra alternativa de tratamiento es el uso de Montelukast en forma diaria. El estudio PREVIA $^{20}$ (PREvention Viral Induced Asthma), demostró que el uso de Montelukast administrado por un año en preescolares de 2 a 5 años con sibilancias gatilladas por virus disminuía el número de exacerbaciones en un $31,9 \%$ comparado con placebo, reduciendo además los síntomas 
diurnos, nocturnos y los días de ausencia escolar; sin embargo, no se encontró diferencias significativas en la necesidad de tratamiento de rescate con agonistas $\beta-2$ adrenérgicos de acción corta, corticoides orales, ni hospitalización comparado con placebo. En la literatura también hay reportes del uso de Montelukast intermitente frente al inicio de síntomas de infección respiratoria ${ }^{21}$. En este caso los pacientes que usaron Montelukast tuvieron una reducción de $28,5 \%$ en el número de visitas no agendadas (médico tratante y Servicio de Urgencia), pero no hubo diferencias en el uso de corticoides orales entre el grupo tratado con Montelukast y el grupo placebo.

En resumen esta Comisión, de acuerdo a la literatura disponible, recomienda realizar una prueba terapéutica durante un período de 3 meses con corticoides inhalados en dosis bajas en todo paciente que presente episodios de obstrucción bronquial muy frecuentes o muy severos independiente del fenotipo clínico y de su condición atópica ${ }^{10,16}$. Como segunda opción se recomienda el uso de Montelukast. La asociación corticoide inhalado más LABA (agonista $\beta-2$ adrenérgico de acción prolongada) es una opción de tratamiento sólo en el mayor de 4 años. Esta mezcla debiera ser indicada por especialista en un preescolar con alta sospecha de asma bronquial en el cual a pesar del uso de corticoides inhalados en dosis adecuadas y con buena adherencia no logra el control de sus síntomas.

La prueba terapéutica requiere de un control estrecho por parte del médico tratante para evaluar si hay mejoría o no. En caso de no haber una respuesta positiva al tratamiento este debe suspenderse. Si la respuesta es favorable el tratamiento igual debe discontinuarse, ya que la mejoría podría no deberse al tratamiento sino a la historia natural favorable de las sibilancias recurrentes a esta edad. La reaparición de los síntomas al suspender el tratamiento y la mejoría al reinstalarlo apoya el diagnóstico de asma. En la Figura 1 se muestra un algoritmo de manejo.

\section{Asma aguda en Preescolares}

La gran mayoría de los preescolares con una exacerbación deberían ser tratados sólo con broncodilatadores inhalados. Los corticoides sistémicos deberían ser reservados para los casos más severos o para los pacientes que requieren hospitalización ${ }^{10}$. A continuación se indica el manejo de las crisis en el Servicio de Urgencia.

Todo preescolar que se presente al Servicio de Urgencia con una crisis de obstrucción bronquial debe ser sometido a tres acciones: evaluación de la gravedad del episodio, intervención terapéutica inmediata y efectiva y finalmente definir el destino del paciente (hospitalización en pediatría o en Unidad de Paciente Crítico (UPC) o alta a su domicilio). Al indicar el alta se debe dejar establecido un seguimiento adecuado, optimizando el tratamiento preventivo y proporcionando herramientas para el manejo precoz de futuras crisis.

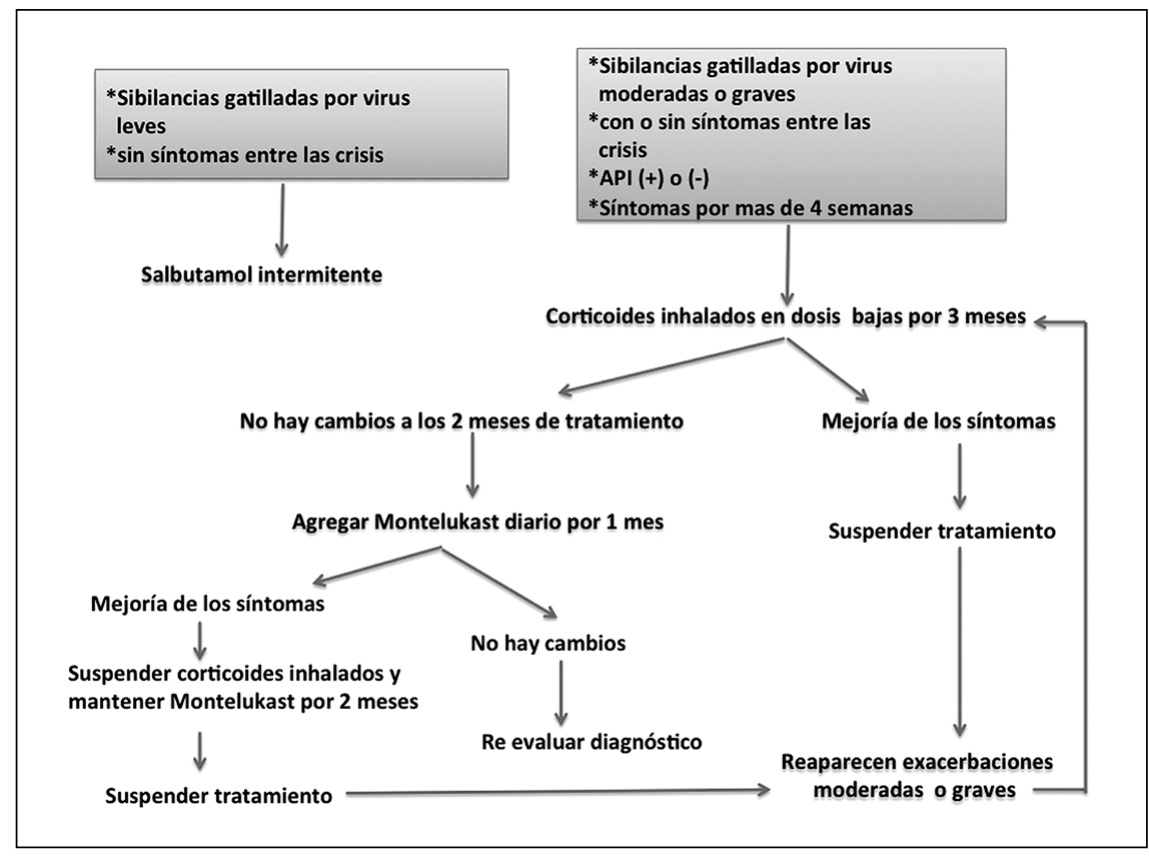

Figura 1. Algoritmo de manejo de las sibilancias recurrentes del preescolar. API = Asthma Predictive Index 5 . 


\section{Evaluación de la gravedad del episodio}

La evaluación inicial debe incluir una breve anamnesis en la cual se indague acerca del inicio del episodio, uso de medicamentos de mantención, uso reciente de $\beta$-2 adrenérgicos o corticoides sistémicos y antecedentes de alergias. Existen algunos factores de riesgo de evolución grave que es útil pesquisar como antecedentes de consultas previas a Servicio de Urgencia, hospitalizaciones, manejo en UPC (unidad de paciente crítico) o necesidad de intubación y uso repetido de corticoides sistémicos en el último año. Al examen físico es importante consignar signos vitales, nivel de conciencia, grado de dificultad respiratoria, presencia de sibilancias, murmullo pulmonar disminuido o ausente, uso de musculatura accesoria y retracciones. No se aconseja realizar en forma rutinaria gasometría arterial o radiografía de tórax. Esta última sólo se justifica si hay sospecha de alguna complicación como atelectasia, neumonía o neumotórax. La Tabla 3 muestra una forma rápida de evaluar la gravedad del episodio, sin embargo, es importante señalar que ninguna puntuación clínica para evaluar el grado de disnea ha sido suficientemente validada en niños ${ }^{22-25}$.

\section{Tratamiento de la exacerbación}

\section{Medidas generales}

Las medidas generales incluyen oxigenoterapia e hidratación. La administración de oxígeno debe lograr saturaciones superiores a $94 \%{ }^{26,27}$. Durante la primera hora de manejo es necesario reevaluar clínicamente al paciente cada 20 a 30 min.

\section{Tratamiento farmacológico}

No existe evidencia científica exclusiva en la edad preescolar que analice el uso de los distintos fármacos que se detallan a continuación ya que la gran mayoría de los estudios publicados incluyen niños de distintas edades (preescolares, escolares $\mathrm{y}$ adolescentes).

\section{Agonistas $\beta$-2 adrenérgicos de acción corta (BAC)}

El salbutamol es el medicamento de elección para manejar la obstrucción bronquial. Se administra en dispositivo de dosis medida (MDI) con espaciador esperando 1 a 2 min entre una y

Tabla 3. Evaluación de la gravedad del episodio de exacerbación aguda

\begin{tabular}{|c|c|c|c|c|}
\hline $\begin{array}{l}\text { Característica } \\
\text { clínica }\end{array}$ & Leve & Moderado & Grave & $\begin{array}{l}\text { Falla respiratoria } \\
\text { inminente }\end{array}$ \\
\hline Estado mental & Normal & Normal o agitado & Generalmente agitado & Somnoliento o confuso \\
\hline Actividad & $\begin{array}{l}\text { Normal } \\
\text { Dificultad respiratoria } \\
\text { con ejercicio }\end{array}$ & $\begin{array}{l}<\text { Actividad } \\
\text { Dificultad respiratoria } \\
\text { en reposo }+\end{array}$ & $\begin{array}{l}<\text { Actividad } \\
\text { Dificultad respiratoria } \\
\text { en reposo }++\end{array}$ & Incapaz de comer \\
\hline $\begin{array}{l}\text { Capacidad para } \\
\text { hablar }\end{array}$ & Normal & Habla frases & Sólo palabras & No puede hablar \\
\hline $\begin{array}{l}\text { Trabajo } \\
\text { respiratorio }\end{array}$ & $\begin{array}{l}\text { Retracción intercostal } \\
\text { mínima o ausente }\end{array}$ & $\begin{array}{l}\text { Retracción intercostal } \\
+/++ \text { y supraesternal }\end{array}$ & $\begin{array}{l}\text { Dificultad respiratoria } \\
++\end{array}$ & $\begin{array}{l}\text { Intensa dificultad } \\
\text { respiratoria en reposo } \\
\text { Uso de toda la muscu- } \\
\text { latura accesoria } \\
\text { Aleteo nasal, } \\
\text { Respiración paradojal }\end{array}$ \\
\hline Auscultación & Sibilancias & $\begin{array}{l}\text { Sibilancias++ en ins- } \\
\text { piración y espiración }\end{array}$ & $\begin{array}{l}\text { Sibilancias audibles } \\
\text { sin fonendoscopio }\end{array}$ & Tórax silente \\
\hline $\begin{array}{l}\mathrm{SaO}_{2} \text { (con aire } \\
\text { ambiental) }\end{array}$ & $>94 \%$ & $91-94 \%$ & $<91 \%$ & $<91 \%$ \\
\hline $\begin{array}{l}\text { Frecuencia de } \\
\text { pulso }\end{array}$ & $<100 / \min$ & $100-180 / \mathrm{min}$ & $>180 / \mathrm{min}$ & $>180 / \mathrm{min}$ \\
\hline Cianosis central & No & No & Posible & Frecuente \\
\hline $\begin{array}{l}\text { Frecuencia } \\
\text { respiratoria }\end{array}$ & Normal & $>40 / \min$ & $>50 / \mathrm{min}$ & Variable \\
\hline
\end{tabular}

No es necesario que estén presentes todos los elementos de una categoría. Los parámetros pueden sobreponerse entre una categoría y otra. 
otra inhalación. Su uso en nebulización (NBZ) se debe considerar en crisis graves ${ }^{28}$. Las dosis recomendadas son las siguientes de acuerdo a la gravedad:

Crisis leve: 4 puffs cada 20 min por $1 \mathrm{~h}^{29-32}$. $\mathrm{Si}$ al cabo de la primera hora de tratamiento el paciente disminuye su frecuencia respiratoria, no tiene signos de dificultad respiratoria y presenta una saturación de $\mathrm{O}_{2}$ igual o mayor de $94 \%$ se considerará una respuesta favorable por lo que se podría indicar el alta luego de un período de observación de $1 \mathrm{~h}$, manteniendo el manejo en forma ambulatoria. Si por el contrario no se obtiene una respuesta adecuada se considerará como crisis moderada.

Crisis moderada: 4 puffs de salbutamol cada $20 \mathrm{~min}$ por $1 \mathrm{~h}^{29-32}$. Si la respuesta no es satisfactoria, repetir esquema. Si al cabo de la segunda hora no hay cambios se debe hospitalizar.

Crisis grave: Preferir NBZ de Salbutamol 0,5 $\mathrm{ml} / 3,5 \mathrm{ml}$ de suero fisiológico $\left(\operatorname{con} \mathrm{O}_{2} \quad 6-8 \mathrm{~L} /\right.$ $\min$, por 6 a $8 \mathrm{~min}$ ), repetir cada $20 \mathrm{~min}$ por 3 veces. Si no hay respuesta adecuada iniciar nebulización continua de Salbutamol $0,3 \mathrm{mg} / \mathrm{kg} / \mathrm{h}^{33}$ y solicitar hospitalización en Intermedio o UPC.

\section{Anticolinérgicos}

Existe evidencia que demuestra beneficio al adicionar anticolinérgicos a los $\mathrm{BAC}$, en las exacerbaciones moderadas y severas ${ }^{33-36}$. No hay estudios en población exclusivamente preescolar, sin embargo, en diferentes guías clínicas se incluye su uso inicial para las exacerbaciones severas $^{29,32}$. En casos graves o frente a falta de respuesta al manejo inicial, adicionar Bromuro de Ipratropio MDI 2 puffs cada 20 min por 3 veces o adicionar a la NBZ con Salbutamol, Bromuro de Ipratropio $0,5 \mathrm{ml}$. No está contemplado su uso después de la primera hora ${ }^{34-37}$.

\section{Glucocorticoides}

En escolares con diagnóstico establecido de asma bronquial se demuestra la utilidad del uso precoz de corticoides sistémicos durante las exacerbaciones ya que reduce la necesidad de hospitalización, el riesgo de recaída y la duración de la hospitalización ${ }^{38,39}$. Sin embargo, la evidencia en preescolares no es suficiente para aconsejar su uso en forma rutinaria ${ }^{40,41}$ debiendo reservarse para pacientes con crisis severa o que requieren hospitalización ${ }^{10}$. Esta Comisión aconseja su uso precoz en pacientes con exacerbación grave o en los casos moderados que no responden al manejo inicial. La vía oral es tan efectiva como la vía intravenosa ${ }^{42}$ : Prednisona: $1 \mathrm{mg} / \mathrm{kg} / \mathrm{día}^{39,40}$ en 1 dosis con un máximo de $40 \mathrm{mg} /$ día por 3-5 días. En casos graves o con falla respiratoria inminente, preferir la vía intravenosa. Se puede utilizar Hidrocortisona $5 \mathrm{mg} / \mathrm{kg} /$ dosis (máximo $200 \mathrm{mg}$ ) cada $6 \mathrm{~h}$ o Metilprednisolona $1 \mathrm{mg} / \mathrm{kg} / \mathrm{dosis}$ (máximo $40 \mathrm{mg}$ ) cada $6 \mathrm{~h}^{39,40}$. Con respecto al uso de glucocorticoides inhalados en vez de glucocorticoides sistémicos en la exacerbación, no hay evidencia consistente que apoye su uso ${ }^{43,44}$.

\section{Sulfato de magnesio}

Se puede utilizar en casos graves, en servicio de urgencia con respuesta incompleta a la terapia convencional y en hospitalizados en UPC. Su uso puede mejorar la función respiratoria y reducir la tasa de hospitalización ${ }^{45,46}$. Se utiliza por vía intravenosa en dosis de $20-50 \mathrm{mg} / \mathrm{kg}$ a pasar en 20 min, máximo $2 \mathrm{~g}^{45-47}$. Es importante monitorizar posibles efectos adversos como bradicardia e hipotensión.

\section{Adrenalina intramuscular}

Está indicada en crisis de asma asociadas a reacción anafiláctica o falla respiratoria inminente en dosis de 0,01 mg im por una vez ${ }^{48}$.

\section{Otras medidas terapéuticas}

Kinesioterapia respiratoria: no está indicada en la etapa inicial de la crisis $^{49}$, pero en fase secretora, en niños con tos inefectiva podría ser de utilidad.

Mucolíticos: no se ha demostrado su utilidad.

Macrólidos: no existe indicación para su uso, excepto cuando el episodio agudo está asociado a una infección por germen atípico ${ }^{50}$.

\section{Criterios de hospitalización}

- Paciente que llegue al Servicio de Urgencia en falla respiratoria inminente o que en su evolución en urgencia, presente esta condición.

- Pacientes que presenten una exacerbación severa.

- Pacientes que después de $2 \mathrm{~h}$ de manejo mantengan exacerbación moderada.

- Pacientes que mantienen requerimiento de $\mathrm{O}_{2}$ $\left(\mathrm{SaO}_{2}<94 \%\right.$ con aire ambiental $)$ después de $2 \mathrm{~h}$ de manejo ${ }^{51}$.

- Crisis asociada a anafilaxia.

- Factores de riesgo: mal acceso a servicios de salud, condiciones sociales muy desfavorables, dificultad en poder seguir un tratamiento supervisado en su domicilio. 


\section{Criterios de ingreso a Unidad de Pacientes \\ Críticos (UPC)}

- Falla respiratoria inminente.

- Exacerbación grave.

- Requerimiento de $\mathrm{FiO}_{2}$ mayor a 0,50, después de $1 \mathrm{~h}$ de manejo.

\section{Criterios de alta de Servicio de Urgencia}

- Saturación arterial de $\mathrm{O}_{2}$ igual o mayor a 94\% con aire ambiental, después de $2 \mathrm{~h}$ de observación.

- Ausencia de signos de dificultad respiratoria.

\section{Indicaciones para el hogar}

- Evitar factores desencadenantes como contaminantes intradomiciliarios (calefacción contaminante, tabaquismo), alergenos a los cuales el niño esté sensibilizado y actividad física entre otros.

- Si requirió corticoides sistémicos completar a lo menos 5 días.

- Salbutamol: continuar su uso 2 puffs cada $4 \mathrm{~h}$ hasta ser controlado por su médico.

- Revisar técnica inhalatoria.

- Plan escrito para manejo precoz en domicilio de futuras exacerbaciones.

- Control por médico especialista en enfermedades respiratorias, para estudio y seguimiento.

\section{Bibliografía}

1.- SEARS M R, GREENE J M, WILLIAM A R, WIECEK E M, TAYLOR D R, FLANNERY E M, et al. A longitudinal, population-based, cohort study of childhood asthma followed to adulthood. N Engl J Med 2003; 349: 1414-22.

2.- PHELAN P D, ROBERTSON C F, OLINSKY A. The Melbourn Asthma Study: 1964-1999. J Allergy Clin Inmunol 2002; 109: 189-94.

3.- MARTÍNEZ F D. What have we learned from the Tucson Children's Respiratory Study. Paediatr Respir Rev 2002; 3: 193-7.

4.- TENERO L, TEZZA G, CATTAZZO E, PIACENTINI G. Wheezing in preschool children. Early Hum Dev 2013; 89 Suppl 3: S13-7.

5.- CASTRO-RODRÍGUEZ J A, HOLBERG C J, WRIGHT A L, MARTÍNEZ F D. A clinical index to define risk of asthma in young children with recurrent wheezing. Am J Respir Crit Care Med 2000; 162: 14036.

6.- MARTÍNEZ F D, WRIGHT A L, TAUSSIG L, HOLBERG C J, HALONEN M, MORGAN W J. Asthma and wheezing in the first six years of life. N Engl J Med 1995; 332: 133-8.
7.- HENDERSON J, GRANELL R, HERON J, SHERRIF A, SIMPSON A, WOODCOCK A, et al. Associations of wheezing phenotypes in the first 6 years of life with atopy, lung function and airway responsiveness in midchildhood. Thorax 2008; 63: 974-80.

8.- SONNAPPA S, BASTARDO C M, WADE A, SAGLANI S, MCKENZIE S A, BUSH A, et al. Symptompattern phenotype and pulmonary function in preschool wheezers. J Allergy Clin Immunol 2010; 126: 519-26.

9.- BRAND P, BARALDI E, BISGAARD, BONER A, CASTRO-RODRÍGUEZ J A, CUSTOVIC A, et al. Definition assessment and treatment of wheezing disorders in preschool children and evidence based approach. Eur Resp J 2008; 32: 1096-110.

10.- BRAND P, CAUDRI D, EBER E, GAILLARD E A, GARCÍA-MARCOS L, HEDLIN G, et al. Classification and pharmacological treatment of preschool wheezing: changes since 2008. Eur Respir J 2014; 43: 1172-7.

11.- PAPADOPOULUS N G, ARAKAWA H, CARLSEN $\mathrm{K}$ H, CUSTOVIC A, GERN J, LEMANSKE R, et al. International consensus on pediatric asthma. Allergy 2012; 67: 976-97.

12.- BUSH A. Diagnosis of asthma in children under five. Prim Care Respir J 2007; 16: 7-15.

13.- BEYDON N, DAVIS S D, LOMBARDI E, ALLEN J L, ARETS H G, AURORA P, et al. An Official American Thoracic Society/European Respiratory Society Statement: Pulmonary Function Testing in Preschool Children. Am J Respir Crit Care Med 2007; 175: 130445.

14.- ROSENFELD M, ALLEN J, ARETS B H, AURORA P, BEYDON N, CALOGERO C, et al. An Official American Thoracic Society Workshop Report: Optimal Lung Function Tests for Monitoring Cystic Fibrosis, Bronchopulmonary Dysplasia and Recurrent Wheezing in Children Less Than 6 years of age. Ann Am Thorac Soc 2013; 10: S1-S11.

15.- KADITIS A G, WINNIE G, SYROGIANNOPULOS GA. Anti-Inflammatory Pharmacotherapy for Wheezing in Preschool Children. Pediatr Pulmonol 2007; 42: $407-$ 20.

16.- CASTRO-RODRÍGUEZ J A, RODRIGO G. Efficacy of inhaled corticosteroids in infants and preschoolers with recurrent wheezing and asthma: a systematic review with meta-analysis. Pediatrics 2009; 123: e519-e525.

17.- GUILBERT T, MORGAN W J, ZEIGER R S, MAUGER D T, BOEHMER S J, SZEFLER S J, et al. LongTerm Inhaled Corticosteroids in Preschool Children at High Risk for Asthma. N Engl J Med 2006; 354: $1985-$ 97.

18.- BACHARIER L, GUILBERT T, ZEIGER R, STRUNK R, MORGAN W, LEMANSKE R, et al. Patient characteristics associated with improved outcomes with use of an inhaled corticosteroid in preschool children at risk for asthma. J Allergy Clin Immunol 2009; 123 : 1077-82. 
19.- DUCHARME F M, LEMIRE C, NOYA F J, DAVIS G $\mathrm{M}, \operatorname{ALOS} \mathrm{N}$, LEBLOND $\mathrm{H}$, et al. Preemptive use of high-dose fluticasone for virus-induced wheezing in young children. N Engl J Med 2009; 360: 339-53.

20.- BISGAARD H, ZIELEN S, GARCÍA-GARCÍA M L, JOHNSTON S, GILLES L, MENTEN J, et al. Montelukast reduces asthma exacerbations in 2 to 5 year old children with intermittent asthma. Am J Respir Crit Care Med 2005; 171: 315-22.

21.- ROBERTSON C, PRICE D, HENRY R, MELLIS C, GLASGOW N, FITZGERALD D, et al. Short course montelukast for intermittent asthma in children. Am J Respir Crit Care Med 2007; 175: 323-9.

22.- BIRKEN C S, PARKIN P C, MACARTHUR C. Asthma severity scores for preschoolers displayed weaknesses in reliability, validity, and responsiveness. J Clin Epidemiol 2004; 57: 1177-81.

23.- CHALUT D S, DUCHARME F M, DAVIS G M. The Preschool Respiratory Assessment Measure (PRAM): A responsive index of acute asthma severity. J Pediatr 2000; 137: 762-8.

24.- REDDEL H K, TAYLOR D R, BATEMAN E D, BOULET L P, BOUSHEY H A, BUSSE W W, et al. An official American Thoracic Society/European Respiratory Society statement: asthma control and exacerbations: standardizing endpoints for clinical asthma trials and clinical practice. Am J Crit Care Med 2009; 180: 59-99.

25.- BEKHOF J, REIMINK R, BRAND P L. Systematic review: Insufficient validation of clinical scores for the assessment of acute dyspnea in wheezing children. Paediatr Respir Rev 2014; 15: 98-112.

26.- RALSTON M, HAZINSKI M F, ZARITSKY A L, SCHEXNAYDER S M, KLEINMAN M E. Pediatric Advance Life Support Course Guide. Dallas, TX: American Heart Association, 2006.

27.- CARROLL W, LENNEY W. Drug therapy in the management of acute asthma. Arch Dis Child Educ Pract Ed 2007; 92 (3): ep82-6.

28.- CASTRO-RODRÍGUEZ J A, RODRIGO G J. Betaagonists through metered-dose inhaler with valved holding chamber versus nebulizer for acute exacerbation of wheezing or asthma in children under 5 years of age: a systematic review with meta-analysis. J Pediatr 2004; 145: $172-7$.

29.- DOUGLAS G, HIGGINS B, BARNES N, BOYTER A, BURGE S, CATES C, et al. British Guideline on the Management of Asthma. Thorax 2008; 63 Suppl 4: $1-121$.

30.- CAMARGO C A JR, RACHELEFSKY, G, SCHATZ M. Managing asthma exacerbations in the emergency department: summary of the National Asthma Education and Prevention Program Expert Panel Report 3 guidelines for the management of asthma exacerbations. J Emerg Med 2009; 37 (2 Suppl): S6-S17.

31.- ORTIZ-ÁLVAREZ O, MIKROGIANAKIS A. Canadian Paediatric Society. Acute Care Committee. Managing the paediatric patient with an acute asthma exacerbation. Paediatr Child Health 2012; 17: 251-62.

32.- PEDERSEN S E, HURD S S, LEMANSKE R F JR, BECKER A, ZAR H J, SLY P D, et al. Global Initiative for Asthma. Global Strategy for the Diagnosis and Management of Asthma in Children 5 Years and Younger. Pediatr Pulmonol 2011; 46: 1-17.

33.- PAPO M C, FRANK J, THOMPSON A E. A prospective, randomized study of continuous versus intermittent nebulized albuterol for severe status asthmaticus in children. Crit Care Med 1993; 21: 1479-86.

34.- ZORC J J, PUSIC M V, OGBORN C J, LEBET R, DUGGAN A K. Ipratropium bromide added to asthma treatment in the pediatric emergency department. Pediatrics 1999; 103: 748-52.

35.- PLOTNICK L H, DUCHARME F M. Combined inhaled anticholinergics and beta 2-agonists for initial treatment of acute asthma in children. Cochrane Database Syst Rev 2000; (4): CD000060. doi: $10.1002 / 14651858$.

36.- RODRIGO G J, CASTRO-RODRÍGUEZ J A. Anticholinergics in the treatment of children and adults with acute asthma: A systematic review with meta-analysis. Thorax 2005; 60: 740-6.

37.- GRIFFITHS B I, DUCHARME F M. Combined inhaled anticholinergics and short-acting beta 2-agonists for initial treatment of acute asthma in children. Cochrane Database Syst Rev 2013; (8): CD000060. doi: 10.1002/14651858.

38.- SMITH M, IQBAL S, ELLIOT T M, EVERARD M, ROWE B H. Corticosteroids for hospitalized children with acute asthma. Cochrane Database Syst Rev 2003; (2): CD002886.

39.- ROWE B H, SPOONER C, DUCHARME F M, BRETZLAFF J A, BOTA G W, et al. Early emergency department treatment of acute asthma with systemic corticosteroids. Cochrane Database Syst Rev 2001; (1): CD002178.

40.- OOMMEN A, LAMBERT P C, GRIGG J. Efficacy of a short course of parent-initiated oral prednisolone for viral wheeze in children aged 1-5 years: Randomized controlled trial. Lancet 2003; 362: 1433-8.

41.- PANICKAR J, LAKHANPAUL M, LAMBERT P C, KENIA P, STEPHENSON T, SMYTH A, et al. Oral prednisolone for preschool children with acute virusinduced wheezing. N Engl J Med 2009; 360: 329-38.

42.- BECKER J M, ARORA A, SCARFONE R J, SPECTOR N D, FONTANA-PENN M E, GRACELY E, et al. Oral versus intravenous corticosteroids in children hospitalized with asthma. J Allergy Clin Immunol 1999; 103: 586-90.

43.- SCARFONE R J, LOISELLE J M, WILEY J F 2ND, DECKER J M, HENRETIG F M, JOFFE M D. Nebulized dexamethasone versus oral prednisone in the emergency treatment of asthmatic children. Ann Emerg Med 1995; 26: 480-6. 
44.- SCHUH S, DICK P T, STEPHENS D, HARTLEY M, KHAIKIN S, RODRIGUES L, et al. High-dose inhaled fluticasone does not replace oral prednisolone in children with mild to moderate acute asthma. Pediatrics 2006; 118: 644-50.

45.- ROWE B H, BRETZLAFF J A, BOURDON C, BOTA $\mathrm{G}$ W, CAMARGO C A Jr. Intravenous magnesium sulfate treatment for acute asthma in the emergency department: a systematic review of the literature. Ann Emerg Med 2000; 36: 181-90.

46.- CHEUK D K, CHAU T C, LEE S L. A meta-analysis on intravenous magnesium sulphate for treating acute asthma. Arch Dis Child 2005; 90: 74-7.

47.- MOHAMMED S, GOODACRE S. Intravenous and nebulized magnesium sulphate for acute asthma: systematic review and meta-analysis. Emerg Med J 2007; 24 :
823-30.

48.- KEMP S F, LOCKEY R F, SIMONS F E. World Allergy Organization ad hoc Committee on Epinephrine in anaphylaxis Epinephrine: the drug of choice for anaphylaxis. A statement of the World Allergy Organization. Allergy 2008; 63: 1061-70.

49.- WALLIS G, PRASAD A. Who needs chest physiotherapy? Moving from anecdote to evidence. Arch Dis Child 1999; 80: 393-7.

50.- WOOD P R, HILL V L, BURKS M L, PETERS J I, SINGH H, KANNAN T R, et al. Mycoplasm pneumoniae in children with acute and refractory asthma. Ann Allergy Asthma Immunol 2013; 110: 328-34.

51.- RODRIGO G, RODRIGO C. A New index for early prediction of hospitalization in patients with acute asthma. Am J Emerg Med 1997; 15: 8-13. 